\title{
Diseño muestral para el estudio del nivel de satisfacción de los usuarios del Departamento de Neonatología de Instituto Especializado Materno Perinatal
}

\author{
Olga Solano, ${ }^{1}$ Doris Gómez ${ }^{1}$, \\ Ana Cárdenas ${ }^{1}, \quad$ Ysabel Adriazola ${ }^{1}$
}

\begin{abstract}
Resumen
En la investigación de Solano, O. y otros [8] se determinaron los factores más importantes que inciden en el nivel de satisfacción de los usuarios del Instituto Especializado Materno Perinatal (IEMP) utilizando el análisis factorial, para lo que fue necesario diseñar el esquema de muestreo. En este contexto el objetivo del presente trabajo es presentar la metodología del diseño muestral, que permitió la generación de los datos posteriormente analizados con el análisis factorial y que se presentarán en una publicación posterior.Se utilizó el muestreo aleatorio estratificado con afijación proporcional [6] y se establecieron los siguientes estratos: Servicio de cuidados intensivos e intermedios, Servicio de alojamiento conjunto de madres adolescentes, servicio de alojamiento conjunto de madres no adolescentes y el servicio de consultorio externo. Las 245 madres participantes en el estudio fueron seleccionadas de cada uno de los cuatro servicios que presta el IEMP, siguiendo el esquema de muestreo estratificado con afijación proporcional. Para el cálculo del tamaño de muestra se consideró un límite para el error de estimación del 6.4\%, a un nivel de confianza del $95 \%$ y la información histórica proporcionada por el IEMP.
\end{abstract}

Palabras Clave: Muestreo aleatorio estratificado, tamaño de muestra, afijación proporcional.

\section{Abstract}

In Solano's investigation and other [8] determined the most important factors that affect in the level of satisfaction of the users of the Specializing Mother Perinatal Institute (IEMP) using the factor analysis, for what was necessary to design the scheme of

${ }^{1}$ UNMSM, Facultad de Ciencias Matemáticas, Lima - Perú. 
sampling. In this context the objective of the present work is present the methodology of the sampling design, the allowed the generation of the information later analyzed with factor analysis and that will appear in a later publication. We considered the stratified random sampling with probabilities proportional to size [6] and the following strata were established : Service of intensive care and intermediate, Service joint housing of teenagers mother, Service joint housing of not teenagers mother and Service of external office. All 245 mothers participants in the study were selected of each of four services that gives the IEMP, following the scheme of stratified random sampling with probabilities proportional to size. For the calculation of the sample size we consider a limit for estimation error of 6,4\%, a confidence level of $95 \%$ and the historical information provided by the IEMP.

Keywords: stratified random sampling, sample size, probabilities proportional to size

\section{Introducción}

Una de las preguntas frecuentes al hacer un estudio de investigación en diversas áreas del conocimiento humano, en particular las ciencias de la salud es: ¿cuál es el tamaño de muestra necesario para el estudio?. La respuesta dependerá de los objetivos, la naturaleza, el alcance y el resultado previsto del estudio, lo cual debe tenerse en cuenta en la fase de la planificación.

Así, podemos citar algunas situaciones de interés. En el trabajo de Lwanga, S. y Lemeshow, S. [7] auspiciado por la OMS (Organización Mundial de la Salud) se presentan cálculos del tamaño de muestra bajo el esquema del muestreo aleatorio simple para estimar la proporción de enfermos. El ministerio de salud desea conocer la prevalencia de tuberculosis entre menores de 5 años. Un investigador de cierto programa nacional de inmunización desea estimar la proporcion de niños que reciben vacunación apropiada durante la infancia. En todos los casos descritos está inherente la obtención del tamaño de muestra.

El Departamento de Neonatología del IEMP actualmente cuenta con los siguientes servicios:

1. Cuidados intensivos / intermedios

2. Alojamiento conjunto madres adolescentes

3. Alojamiento conjunto madres no adolescentes

4. Consultorio externo (pediatría social)

que serán considerados como estratos.

En el servicio de cuidados intensivos/intermedios se encuentran hospitalizados los bebés prematuros, que nacen con bajo peso, muchas veces con $500 \mathrm{gr}$, o algunos con problemas de salud y tienen que quedarse allí hasta conseguir el peso requerido y logren estabilizar 


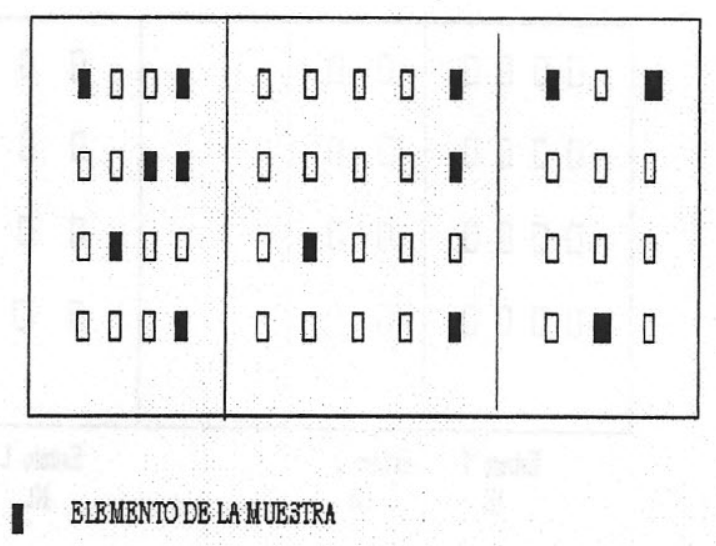

Figura 1: Esquema gráfico del Muestreo estratificado población con tres estratos

su salud, no necesariamente estos bebés han nacido en la maternidad, en algunos casos son trasladados de centros de salud de Lima o provincia, porque no tienen el instrumental necesario para atender al bebé. El servicio de alojamiento conjunto de madres adolescentes atiende a madres parturientas que tienen entre 13 y 19 años de edad. Si el parto es normal en promedio permanecen hospitalizadas de 1 a 3 días y si el parto es por cesárea la permanencia es de 3 a 5 días.

En el servicio de alojamiento conjunto de madres no adolescentes se encuentran las madres parturientas de 20 y más años de edad.

El servicio de consultorio externo (pediatría social) atiende a los bebés que han sido dados de alta en el servicio de cuidados intensivos / intermedios y que acuden a este servicio para su control mensual.

A continuación presentamos la teoría del muestreo estratificado y la determinación del tamaño de la muestra para el estudio de los niveles de satisfacción en los servicios del Departamento de Neonatología.

\section{Diseño Muestral : Muestreo Estratificado}

Una muestra estratificada es obtenida mediante la separación de los elementos en grupos llamados estratos, y la selección posterior de una muestra aleatoria de cada estrato, por ejemplo, la Figura 1 muestra un esquema gráfico del muestreo estratificado de una población con tres estratos.

El procedimiento de selección de una muestra estratificada consiste en:

1. Generalizando para $L$ estratos independientes $(L>1)$ tenemos:

$N_{i}$ : Tamaño del estrato $i,(i=1,2, \ldots, L)$

$N=\sum_{i=1}^{L} N_{i}$ : Tamaño de la población. 


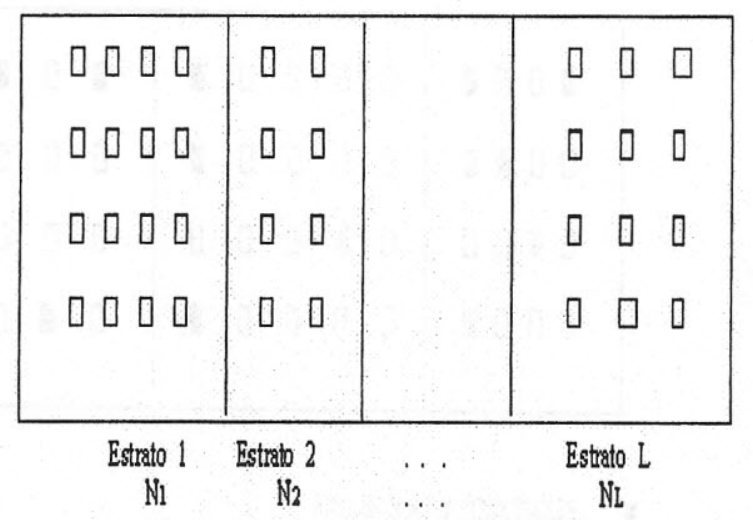

Figura 2: Estratificación de una población

(ii) Seleccionar una muestra aleatoria independientemente de cada estrato, de tal manera que el tamaño total de la muestra es:

$$
n=\sum_{i=1}^{L} n_{i}
$$

donde :

$n_{i}$ es el tamaño de la muestra en el estrato $i$.

Existen tres criterios que deben tenerse presente cuando se está planeando utilizar el muestreo estratificado.

1. Los estratos deben formarse de tal manera que se garantice la independencia entre estratos. Es decir, los estratos deben ser completamente independientes en el proceso de selección y estimación.

2. Las mediciones dentro de los estratos deben ser homogéneas (baja variabilidad).

3. Las mediciones entre estratos deben ser heterogéneas (alta variabilidad).

\subsection{Estimación de una proporción poblacional}

Si lo que se desea es estimar la proporción poblacional $P$, de una característica determinada, se usa

$$
\begin{aligned}
\hat{P}_{e s t} & =\frac{1}{N}\left(N_{1} p_{1}+N_{2} p_{2}+\ldots+N_{L} p_{L}\right) \\
& =\frac{1}{N} \sum_{h=1}^{L} N_{h} p_{h}=\sum_{h=1}^{L} W_{h} p_{h}
\end{aligned}
$$

donde:

$$
W_{h}=\frac{N_{h}}{N}
$$


$p_{h}$ : Proporción de individuos con la característica de interés en el estrato $h$.

$$
q_{h}=1-p_{h}
$$

1. Sólo si los $p_{h}$ son muy diferentes de estrato a estrato, vale la pena estratificar.

2. Si $0,2 \leq P_{h} \leq 0,8$ para todo $h$, no conviene usar los estratos.

La varianza estimada del estimador de la proporción poblacional es

$$
\hat{V}\left(\hat{P}_{e s t}\right)=\frac{1}{N^{2}} \sum_{h=1}^{L} N_{h}^{2}\left(1-f_{h}\right)\left(\frac{p_{h} q_{h}}{n_{h}-1}\right)
$$

El límite para el error de estimación, de la proporción poblacional, es

$$
2 \sqrt{\hat{V}\left(\hat{P}_{e s t}\right)}=2 \sqrt{\frac{1}{N^{2}} \sum_{h=1}^{L} N_{h}^{2}\left(1-f_{h}\right)\left(\frac{p_{h} q_{h}}{n_{h}-1}\right)}
$$

El intervalo de confianza para la proporción de la población es

$$
\hat{P}_{e s t} \pm Z \sqrt{\hat{V}\left(\hat{P}_{e s t}\right)}
$$

donde

$$
\hat{P}_{e s t}=\sum_{h=1}^{L} W_{h} p_{h}
$$

$Z$ : es la abscisa de la distribución normal.

\subsection{Selección del tamaño de muestra para estimar proporciones}

Para estimar una proporción poblacional, primero indicamos qué tanta información deseamos obtener mediante la especificación del tamaño del límite para el error de estimación. La fórmula para el tamaño de muestra $n$ (para un límite del error de estimación) está dada por

$$
n=\frac{\sum_{h=1}^{L} N_{h}^{2} p_{h} q_{h} / W_{h}}{N^{2} D+\sum_{h=1}^{L} N_{h} p_{i} q_{i}}
$$

donde :

$W_{h}$ es fracción de observaciones asignadas al estrato $h$ 
$D=\frac{B^{2}}{4}$ para estimar la proporción poblacional $P_{\text {est }}$

$2 \sqrt{V\left(\hat{P}_{\text {est }}\right)}=B$ (la estimación de $P_{\text {est }}$ debe estar dentro de $B$ unidades de la media poblacional, con una probabilidad aproximadamente igual a 0.95 )

\subsection{Afijación del tamaño de muestra en cada estrato}

El objetivo del diseño de encuesta por muestreo es proporcionar estimadores con varianzas pequeñas al menor costo posible. Después de elegir el tamaño de muestra $n$, existen muchas maneras para dividir $n$ entre los tamaños de muestra de los estratos individuales $n_{1}, n_{2}, \ldots$, $n_{L}$. Cada división puede originar una varianza diferente para la media muestral.

Por lo que nuestro objetivo es usar una afijación que presente una proporción especificada de información a un costo mínimo.

De acuerdo a este objetivo, el mejor esquema de afijación está influido por tres factores:

1. El número total de elementos en cada estrato

2. La variabilidad de las observaciones dentro de cada estrato.

3. El costo por obtener una observación de cada estrato.

A continuación se presenta un resumen de las metodologías más conocidas para determinar en la muestra el tamaño de cada estrato.

2.3.1. Afijación aproximada que minimiza el costo para el valor fijo de $V\left(\bar{y}_{e s t}\right)$ o que minimiza $V\left(\bar{y}_{\text {est }}\right)$

para un costo fijo:

$$
\begin{aligned}
n_{h}= & n\left(\frac{N_{h} \sigma_{h} / \sqrt{c_{h}}}{N_{1} \sigma_{1} / \sqrt{c_{1}}+N_{2} \sigma_{2} / \sqrt{c_{2}}+\ldots+N_{L} \sigma_{L} / \sqrt{c_{L}}}\right) \\
& =n\left(\frac{N_{h} \sigma_{h} / \sqrt{c_{h}}}{\sum_{h=1}^{L} N_{h} \sigma_{h}^{2}}\right)
\end{aligned}
$$

donde:

$N_{h}$ : denota el tamaño del $h$-ésimo estrato,

$\sigma_{h}^{2}$ : denota la varianza poblacional para el $h$-ésimo estrato

$c_{h}$ : denota el costo para obtener una observación individual

del $h$-ésimo estrato. 


\subsubsection{Afijación de Neyman}

En algunos problemas de muestreo estratificado, el costo por obtener información es el mismo para todos los estratos. Si los costos son desconocidos, podríamos suponer que los costos por observación son iguales. Si $c_{1}=c_{2}=c_{3}$, entonces los términos de costo se cancelan en la ecuación (2.5) y se obtiene

$$
n_{h}=n\left(\frac{N_{h} \sigma_{h}}{\sum_{h=1}^{L} N_{h} \sigma_{h}}\right)
$$

este método de seleccionar $n_{1}, n_{2}, \ldots, n_{h}$ se denomina afijación de Neyman.

\subsubsection{Afijación proporcional}

Además de encontrar costos iguales, en algunas ocasiones resultan las mismas varianzas, $\sigma_{1}^{2}, \sigma_{2}^{2}, \ldots, \sigma_{L}^{2}$. En tal caso se cancelan las $\sigma_{h}$ en la ecuación (2.6) y

$$
n_{h}=n\left(\frac{N_{h}}{\sum_{h=1}^{L} N_{h}}\right)=n\left(\frac{N_{h}}{N}\right)
$$

Este método de asignación de la muestra a los estratos es llamado afijación proporcional porque los tamaños de muestra $n_{1}, n_{2}, \ldots, n_{L}$ son proporcionales a los tamaños de los estratos $N_{1}, N_{2}, \ldots, N_{L}$.

La asignación proporcional puede y suele utilizarse cuando los costos y las varianzas de los estratos no son iguales.

\section{Resultados}

En esta sección se presentan los resultados de la investigación usando el método con afijación proporcional.

\section{Muestra piloto}

La encuesta piloto se realizó entre los meses de Enero a Marzo del año 2005 con la finalidad de detectar los posibles errores en el instrumento de medición diseñado para el estudio. La aplicación de las encuestas a las madres estuvo a cargo de los profesionales del Instituto de Investigación de la Facultad de Ciencias Matemáticas (UNMSM), quienes en ningún caso realizan actividades de atención de los pacientes.

Para estudiar la confiabilidad del Instrumento de medición, la cual se refiere a la consistencia de la medición, se utilizó el coeficiente de confiabilidad alfa de Cronbach cuyo valor fue de 0,92 , indicando una confiabilidad elevada y muy buena. 
Después de la validación el instrumento final quedo diseñado de la siguiente manera:

1. Parte I : Aspectos generales: Características de las madres con bebés hospitalizados en los servicios de Cuidados Intensivos e Intermedios del IEMP durante el periodo de estudio.

2. Parte II: Aspectos específicos sobre nivel de satisfacción, en los que se recogía opiniones del usuario, desde "Totalmente en desacuerdo" (puntaje 1) hasta "Totalmente de acuerdo." (puntaje 20).

\section{Muestra definitiva}

Los servicios de :

1. Cuidados Intensivos / Intermedios

2. Alojamiento Conjunto Madres Adolescentes

3. Alojamiento Conjunto Madres No Adolescentes

4. Consultorios Externos

del Departamento de Neonatología del IEMP fueron tratados como estratos.

1. La unidad informante en todos los casos será la madre.

2. La información histórica sobre el número promedio de pacientes atendidos mensualmente en cada servicio en el año 2004 fue la siguiente :

Como deseamos conocer el nivel de satisfacción de los usuarios Sobre la calidad de servicio que brinda el Departamento de Neonatología del IEMP, optamos por utilizar el muestreo estratificado con afijación proporcional al tamaño de los estratos [6] a fin captar el grado de satisfacción en cada una de los servicios.

Así, para:

$p_{i}=0,5(\mathrm{i}=1,2,3,4):$ proporción de madres completamente satisfechas sobre la calidad del Servicio recibido del $i$ - ésimo servicio del Departamento de Neonatología ;

$$
\begin{gathered}
W_{i}=\frac{N_{i}}{N}, i=1,2,3,4 \\
N=3134, W_{1}=\frac{767}{3134}, W_{2}=\frac{367}{3134}, W_{3}=\frac{1100}{3134}, W_{4}=\frac{900}{3134}, D=\frac{E^{2}}{4} ;
\end{gathered}
$$

Se escoge $E=0,064$, límite para el error de estimación con un nivel de confianza del $95 \%$.

Usando (2.4) el tamaño de muestra fue:

$$
n=\frac{767^{2}(0,5)(0,5) /\left(\frac{767}{3134}\right)+367^{2}(0,5)(0,5) /\left(\frac{367}{3134}\right)+1100^{2}(0,5)(0,5) /\left(\frac{1100}{3134}\right)+900^{2}(0,5)(0,5) /\left(\frac{1100}{3131}\right)}{3134^{2}\left(\frac{0,064}{4}\right)+767(0,5)(0,5)+367(0,5)(0,5)+1100(0,5)(0,5)+900(0,5)(0,5)}
$$




\begin{tabular}{|l|l|l|}
\hline Nro & Servicios & $\begin{array}{l}\text { Promedio } \\
\text { (Ni) }\end{array}$ \\
\hline 1 & $\begin{array}{l}\text { Cuidados intensivos/ Cuidados Inter- } \\
\text { medios }\end{array}$ & 767 \\
\hline 2 & $\begin{array}{l}\text { Servicio de alojamiento Conjunto - } \\
\text { Madres Adolescentes }\end{array}$ & 367 \\
\hline 3 & $\begin{array}{l}\text { Servicio de alojamiento Conjunto - } \\
\text { Madres No Adolescentes }\end{array}$ & 1100 \\
\hline 4 & $\begin{array}{l}\text { Consultorios Externos (Pediatria So- } \\
\text { cial) }\end{array}$ & 900 \\
\hline & Total (N) & 3134 \\
\hline
\end{tabular}

Cuadro 1: Número promedio de Pacientes atendidos mensualmente - Año 2004 . Fuente: IEMP

$n=245$. Es decir, el tamaño de muestra total fue de 245 unidades informantes.

Estas 245 unidades, según la metodología de muestreo ęstratificado con afijación proporcional, usando (2.7) se repartirán proporcional al tamaño de cada estrato de la población, como se muestra a continuación.

a) Para el servicio de cuidados intensivos / intermedios:

$n_{1}=n\left(\frac{N_{h}}{N}\right)=245\left(\frac{767}{3134}\right)=60$ unidad informantes.

Es decir en el servicio de cuidados intensivos/intermedios seleccionamos a 60 madres que tienen a sus bebés hospitalizados en ese servicio.

Asimismo:

b) Para el servicio de alojamiento conjunto de madres adolescentes:

$n_{2}=n\left(\frac{N_{2}}{N}\right)=245\left(\frac{367}{3134}\right)=29$ unidades informantes.

c) Para el servicio de alojamiento conjunto de madres no adolescentes:

$n_{3}=n\left(\frac{N_{3}}{N}\right)=245\left(\frac{1100}{3134}\right)=86$ unidades informantes.

d) Para el servicio de consultorio externo:

$n_{4}=n\left(\frac{N_{4}}{N}\right)=245\left(\frac{900}{3134}\right)=70$ unidades informantes.

Finalmente, la distribución de la muestra según los diferentes servicios del Departamento de Neonatologia se muestra a continuación:

El muestreo aleatorio estratificado determinó seleccionar a 245 madres informantes, distribuidas de la siguiente manera: 60 madres en el servicio de cuidados intensivos/intermedios, 29 madres en el servicio de alojamiento conjunto de madres adolescentes, 86 madres en el servicio de alojamiento conjunto de madres no adolescentes y 70 madres en el servicio de consultorio externo. 


\begin{tabular}{|l|l|}
\hline Servicios & $n_{i}=n\left(\frac{N_{i}}{N}\right)$ \\
\hline Cuidados intensivos/intermedios & 60 \\
\hline Alojamiento conjunto de madres adolescentes & 29 \\
\hline Alojamiento conjunto de madres no adolescentes & 86 \\
\hline Consultorio externo (pediatria social) & 70 \\
\hline Total (n) & 245 \\
\hline
\end{tabular}

Cuadro 2: Tamaño de muestra requerido para cada servicio

\section{Discusión y Comentarios}

Siguiendo el esquema de muestreo estratificado con afijación proporcional. se cálculo del tamaño de muestra y se considero un límite para el error de estimación del $6.4 \%$, a un nivel de confianza del $95 \%$ y la información histórica proporcionada por el IEMP. Las 245 madres participantes en el estudio fueron seleccionadas de cada uno de los cuatro servicios que presta el IEMP,

\section{Referencias}

[1] BERNÉ, C., MÚGICA, J. M. y YAGÜE, M. J. La gestión estratégica y los conceptos de calidad percibida, satisfacción del cliente y lealtad, Economía Industrial, 1996.

[2] BIGNÉ E.; MOLINER, M. Y SÁNCHEZ, J. Calidad y Satisfacción en los Servicios Hospitalarios y periféricos. Investigación y Marketing, 1997.

[3] OLIVER, R.L. A Cognitive Model of Antecedents and Consequences of satisfaction Decisions, Journal of Marketing Research, vol XVII, pgs. 460-469, 1980.

[4] ORGANIZACIÓN PANAMERICANA DE LA SALUD. Investigaciones sobre servicios de salud: una antología, Publicación Científica № 534, Washington, D.C., 1992.

[5] RUST, R.T. y OLIVER, R. L. Service Quality: Insights and managerial Implications from the Frontier, en Service Quality; New Directions in Theory and Practice, Rust y Oliver eds., Sage Publications, 1994.

[6] SCHEAFFER, R., MENDENHALL, W. y OTT, L. Elementos de muestreo. Grupo Editorial Iberoamérica. México, 1987.

[7] LWANGA, S. Y, LEMESHOW S. Determinación del tamaño de las muestras en los estudios sanitarios. Manual práctico. Organización Mundial de la Salud. Ginebra, 1991. 
[8] SOLANO, O. y otros . Estudio de satisfacción del servicio público de salud usando métodos multivariantes. Caso: Instituto Materno Perinatal de Lima. Informe final presentado al Instituto de Investigación de la Facultad de Ciencias Matemáticas. UNMSM. Lima. Perú, 2005. 